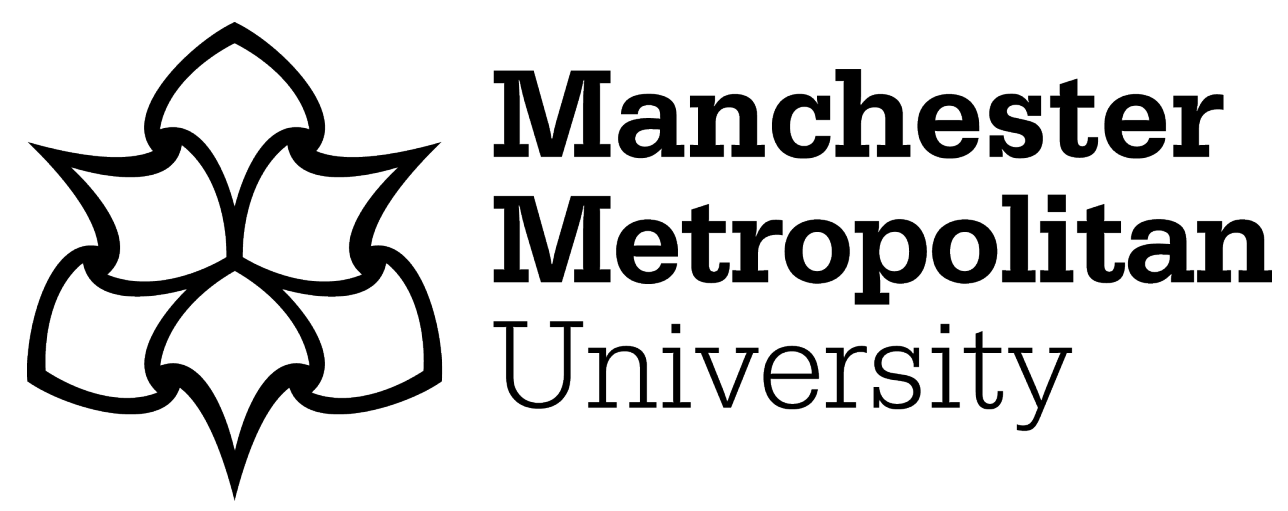

Lynch, Stephen ORCID logoORCID: https://orcid.org/0000-0002-4183-5122, Borresen, Jon, Roach, Paul, Kotter, Mark and Slevin, Mark (2020) Mathematical Modeling of Neuronal Logic, Memory and Clocking Circuits. International Journal of Bifurcation and Chaos, 30 (01). p. 2050003. ISSN 0218-1274

Downloaded from: https://e-space.mmu.ac.uk/625119/

Version: Accepted Version

Publisher: World Scientific Pub Co Pte Lt

DOI: https://doi.org/10.1142/s0218127420500030

Please cite the published version 


\title{
MATHEMATICAL MODELING OF NEURONAL LOGIC, MEMORY AND CLOCKING CIRCUITS
}

\author{
Stephen Lynch and Jon Borresen \\ Department of Computing and Mathematics, \\ Manchester Metropolitan University, Manchester M1 5GD, \\ United Kingdom, \\ s.lynch@mmu.ac.uk, j.borresen@mmu.ac.uk \\ Paul Roach \\ Department of Chemistry, \\ Loughborough University, Loughborough, LE11 3TU, \\ United Kingdom, \\ p.roach@lboro.ac.uk \\ Mark Kotter \\ Department of Clinical Neurosciences, \\ University of Cambridge, Cambridge, CB2 0SZ, \\ United Kingdom, \\ mrk25@cam.ac.uk \\ Mark Slevin \\ Department of Life Sciences, \\ Manchester Metropolitan University, Manchester, M1 5GD, \\ United Kingdom, \\ m.a.slevin@mmu.ac.uk
}

\begin{abstract}
The differential equations used to model biological neurons and the chemical kinetics involved in synaptic excitation and inhibition have been well-established for a number of decades. For the first time, this paper presents mathematical and computational models of a neuronal binary oscillator half-adder, a neuronal Set-Reset (SR) flip-flop and a simple neuronal clocking circuit, which have all been shown to be noise resistant. In modern computers, the half-adder is the basic component to perform logic, the SR flip-flop is used to store memory and clocking circuits are used to synchronize components in parts of the computer. These novel circuits will provide the world with neuronal assays that can measure the functionality of the neurons and hence provide more information than is available with current technology. The authors are not proposing to build conventional computers with these components (they would be too slow to be practical) but the simple circuits could be used to measure the functionality of diseased circuits which are subjected to certain drugs. Neurological conditions research into Alzheimer's disease, epilepsy and Parkinson's disease, for example, would all benefit from this research. These assays for neuronal degradation could have major implications for the National Center for the Replacing, Reducing and Refining the use of animals for scientific testing - otherwise known as the NC3R agenda.
\end{abstract}


Keywords: Assay, binary oscillator, CMOS, half-adder, Josephson junction, memristor, neurological disorders, neuron, noise resistant, optical oscillator, set reset flip-flop, superconducting nanowire, threshold oscillator

\section{Introduction}

In 2018, the UK government working with a panel of experts from academia and business published the Blackett Review [Peplow, 2018], an article written by experts in the field for non-experts. The article highlights the importance of simulation utilizing computational and mathematical modeling of complex systems in both the public and private sectors. The review demonstrates how modeling increases efficiency and quality whilst reducing the need for physical prototypes and testing. The manufactured products resulting from the research presented in this paper will be neuronal assays. These in vitro assays are simulated as part of the design process and this should help greatly with the creation of a physical prototype. On the micro-level (the smallest components), neurons and synapses are modeled using well-established differential equations. On the meso-level (how the components are connected together), novel neuronal circuitry is described in this paper for the first time. On the macro-level (properties of the system as a whole), neuronal circuits will behave as half adders, SR flip-flops and simple clocking circuits - the basic components of modern computers.

In 2009, Borresen and Lynch [Borresen, 2009; Lynch, 2012] first proposed the principle of binary oscillator computing. Shortly afterwards, the authors demonstrated binary threshold oscillator logic including circuits for a half adder, a full adder, a seven-input full adder and a $2 \times 2$ bit binary multiplier [Borresen, 2012]. They were also able to demonstrate a working threshold oscillator SR flip-flop which was extremely noise resistant. The authors were able to construct simple mathematical models to demonstrate a binary oscillator half-adder using biophysically simplified coupled Fitzhugh-Nagumo equations using a sigmoidal transfer function which is often used in neural networks. Figure 1 shows the schematics and input and output for binary half adders which are the basic building blocks for logic operations in modern computers. Figure 1(a) shows the logic circuit for a binary half-adder comprising of two inputs A and B, two outputs $\mathrm{S}$ (sum) and $\mathrm{C}$ (carry), an exclusive or (XOR) gate and an AND gate. Figure 1(b) shows the truth table for the half-adder which is used to add two bits (A and B) together. Figure 1(c) shows a schematic of a binary oscillator half adder comprising of two inputs $\mathrm{A}$ and $\mathrm{B}$, two outputs $\mathrm{S}$ and $\mathrm{C}$, and two oscillators $\mathrm{O} 1$ (acts as an XOR gate for the sum) and O2 (acts as an AND gate for the carry). Note that in our simulations, oscillator $\mathrm{O} 1$ has a lower threshold than oscillator O2. Figure 1(d) shows the time series of the binary oscillator half-adder. Comparison with the truth table in Figure 1(b) shows that it is functioning correctly. Output $\mathrm{S}$ corresponds to units and output $\mathrm{C}$ represents tens, an oscillation represents a binary one and no oscillation is zero. Looking down the columns, if A and B are off then $S$ and $C$ are not oscillating and there is no output $(0+0=0)$. If either $\mathrm{A}$ or $\mathrm{B}$ is oscillating then the threshold of oscillator $\mathrm{O} 1$ is reached and that oscillator fires, whereas the threshold of oscillator $\mathrm{O} 2$ is not reached and so does not fire $(0+1=1$ or $1+0=1)$. Looking at the final column, if both inputs are on, then $S$ and $C$ are oscillating, the thresholds of both oscillators are achieved, both start to oscillate but oscillator O2 inhibits oscillator O1, preventing it from firing $(1+1=10)$.

Figure 2 shows the schematics and input and output for binary Set Reset (SR) flip-flops which are used to store memory in modern computers. Figure 2(a) shows the logic circuit for a binary SR flip-flop comprising of two inputs $\mathrm{R}$ and $\mathrm{S}$, two outputs $\mathrm{Q}$ and $\bar{Q}$, and two NOR gates. Figure $2(\mathrm{~b})$ shows the truth table for the SR flip-flop. Figure 2(c) shows a schematic of a binary oscillator SR flip-flop comprising of two inputs $\mathrm{R}$ and $\mathrm{S}$, two outputs $\mathrm{Q}$ and $\bar{Q}$, and two oscillators $\mathrm{O} 1$ and $\mathrm{O} 2$ which act as a pair of cross-coupled NOR or NAND gates. Figure 2(d) shows the time series of the binary oscillator SR flip-flop. Comparison with the truth table in Figure 2(b) shows that it is functioning correctly.

There are two major advantages in using threshold oscillators over transistor-based logic and memory circuits. In the first case, for logic circuits, the processing power of threshold oscillator circuits can be doubled with a linear increase in components. Much like the human brain, the power comes from the high 


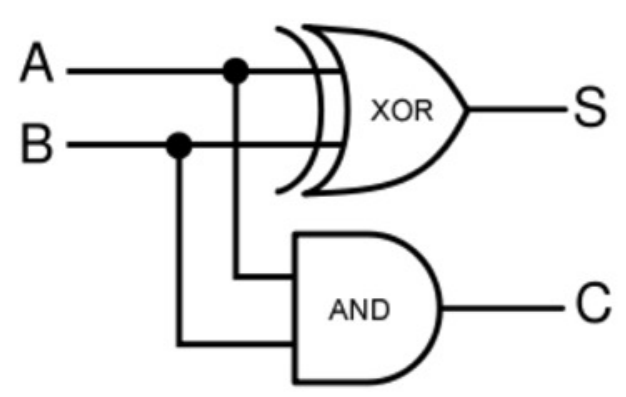

\begin{tabular}{|c|l|l|l|l|}
\hline A & 0 & 0 & 1 & 1 \\
\hline B & 0 & 1 & 0 & 1 \\
\hline S & 0 & 1 & 1 & 0 \\
\hline C & 0 & 0 & 0 & 1 \\
\hline
\end{tabular}

(a)

(b)

A

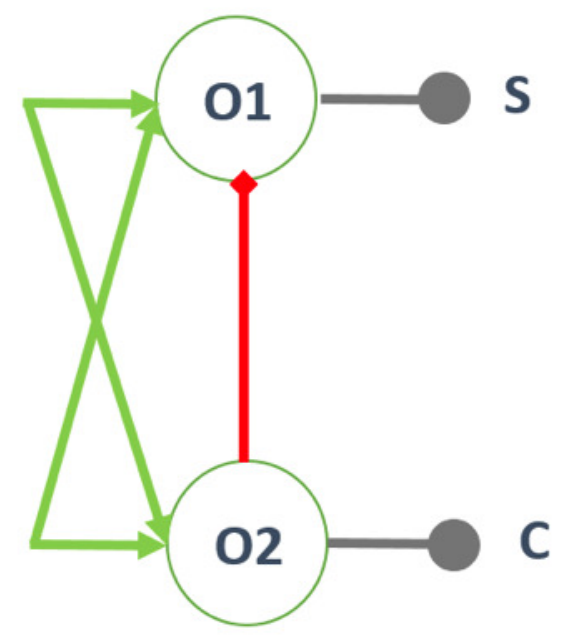

(c)
A

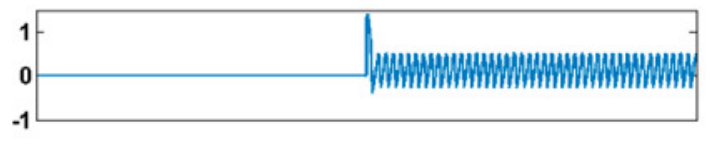

B

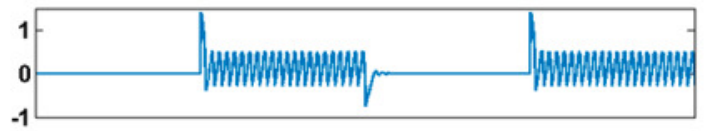

$s$

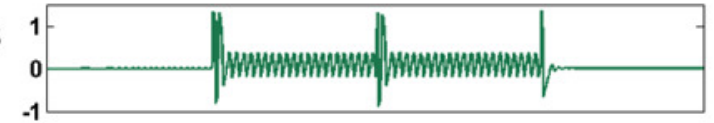

C

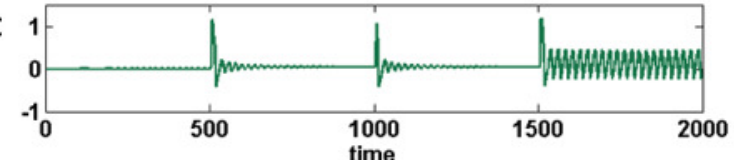

(d)

Fig. 1. Binary half adders. (a) Schematic of a logic circuit for a binary half adder, where A and B are inputs, S (sum) and C (carry) are outputs. (b) Truth table for a binary half adder. (c) Schematic of a binary oscillator half adder. The green arrows depict excitatory connections and the red arrow denotes an inhibitory connection. (d) Time series of the binary threshold oscillator half adder based on biophysically meaningless Fitzhugh-Nagumo models. The device is functioning as a half-adder (compare with the truth table in (b)).

connectivity. Transistor technology requires an exponential growth in the number of components, hence the need for chips containing billions of transistors. Secondly, for SR flip-flop circuits, threshold oscillator switches can be caused by ballistic propagation - where a single pulse can cause a switch (see Figure 2(d)). Using transistor-based SR flip-flops costs far more power as the whole line has to be charged to cause a switch (see Figure 2(a)).

There are potentially six avenues of research for binary oscillator computing and we will now briefly introduce each avenue in turn providing a number of references for the interested reader:

(1) Transistor-based threshold oscillators. These oscillators could be employed like those used in many neuromorphic computers being built today [Indiveri, 2011; Merolla, 2014; Ohno, 2011; Zhou, 2015]. Indiveri et al. [Indiveri, 2011] describe the most common transistor-based Complementary MetalOxide-Semiconductor (CMOS) circuitry and techniques used for hardware implementation of spiking neurons for a large variety of applications. As yet, there are no CMOS circuits for binary oscillator computing and this remains an open avenue of research.

(2) Josephson junction (JJ) neurons. JJs are superconducting threshold oscillators and unsurprisingly they can be used to model neurons [Lynch, 2013] with the added advantages that they are much faster oscillators than biological neurons; the signal processing properties are closely aligned to biological 


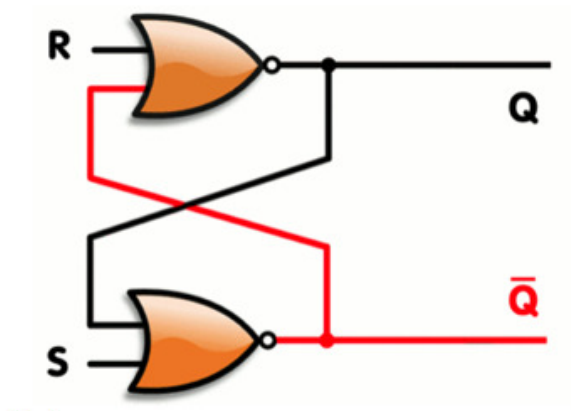

(a)

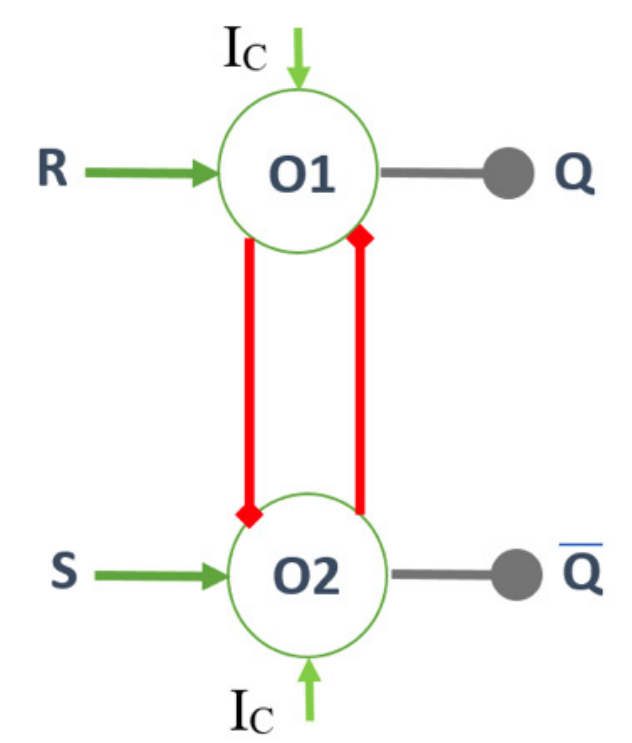

(c)

\begin{tabular}{|c|c|c|c|}
\hline$R$ & 0 & 1 & 0 \\
\hline$S$ & 0 & 0 & 1 \\
\hline$Q$ & 0 & 1 & 0 \\
\hline $\bar{Q}$ & 1 & 0 & 1 \\
\hline
\end{tabular}

(b)

R

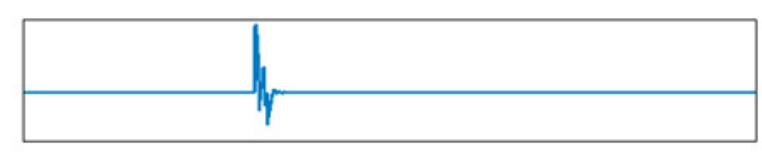

$\mathrm{S}$

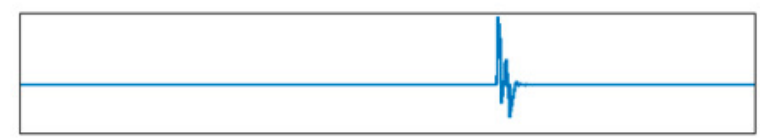

Q
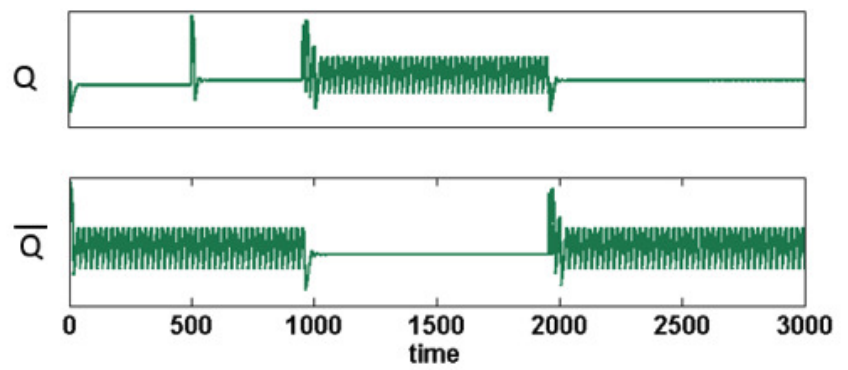

(d)

Fig. 2. Set Reset (SR) flip-flops. (a) Schematic of a logic circuit for an SR flip-flop, where R and S are inputs and $\mathrm{Q}$ and $\bar{Q}$ are outputs, where $\bar{Q}$ is not Q. (b) Truth table for a binary SR flip-flop. (c) Schematic of a binary oscillator SR flip-flop comprising two inputs $\mathrm{R}$ and $\mathrm{S}$, two threshold oscillators with inhibitory connections between them and two outputs $\mathrm{Q}$ and $\bar{Q}$. Note that oscillators O1 and O2 act as a pair of cross-coupled NOR or NAND gates. (d) Time series of the binary threshold oscillator SR flip-flop based on biophysically meaningless Fitzhugh-Nagumo models. The device is functioning as an SR flip-flop (compare with the truth table in (b)). Note that line charging is required in (a) but only a single pulse is required to cause a switch for an oscillator SR flip-flop.

neurons; large arrays can be packed into small volumes; they operate in low noise environments with temperatures of a few Kelvin and finally, the power dissipation is very low (in the order of $10^{-9} \mathrm{~W}$ per junction on average). JJs are natural threshold oscillators so it should come as no surprise that simple circuits can be built that mimic the functionality of neurons, see references [Crotty, 2010; Dana, 2006; Mizugaki, 1993; Mukhanov, 1988], for example. More recently, scientists at HYPRES, Inc. (www.hypres.com) invented Rapid Single Flux Quantum (RSFQ) logic [Filippov, 2012] which used over-damped JJs and two-junction interferometers to pass, store and process data as single flux quanta. In 2017, Segall et al. [Segall, 2017] built a physical circuit where JJ neurons synchronize in picoseconds and more recently they have simulated JJ neuron half-adder and SR flip-flop circuits, this research will be published at a later date. Interested readers should consult references [Fillipov, 2011; Josephson, 1962, 1974; Mukhanov, 1987, 2011; Ryazanov, 2012] for more information.

(3) Superconducting nanowires. In June 2019, Toomey, Segall and Berggren [Toomey, 2019] proposed the use of superconducting nanowires as a platform for the development of an artificial neuron. The 
authors were able to demonstrate multiple neuron behaviors using electrothermal circuit simulations computed in LTSpice. As well as modeling excitation and inhibition, the authors were also able show how a single neuron could inhibit four target neurons thus demonstrating the capability of fanout. Superconducting nanowires can interface with both CMOS and JJs. The authors are currently attempting to make physical circuit prototypes, and the nanowire neuron is a promising candidate for low-power artificial neural networks as well as binary oscillator computing.

(4) Memristors and neuristors. In 1971, Leon Chua proved the existence of the memristor [Chua, 1971] and thirty seven years later a team at HP Laboratories [Strukov, 2008] declared that they had built a memristive device. Memristors can be used to form neuristors (built using two nanoscale Mott memristors) which are simple devices that can capture the essential characteristics of a neuron [Pickett, 2013]. Nanoscale memristors can act as synapses in neuromorphic systems [Jo, 2010]. Furthermore, memristor nanodevices can act as synapses to add dynamic learning to neuromorphic networks implemented in CMOS/memristor hybrids based on a crossbar lattice network [Afifi, 2009]. It is claimed that memristors can be used to provide high connectivity between electronic neurons - where one neuron can connect to a thousand other neurons. For more detail on memristors the reader is directed to references [Chua, 2013; Kim, 2012; Linn, 2014]. More recently, Papandroulidakis et al. [Papandroulidakis, 2019] have given a practical implementation of memristor-based threshold logic gates.

(5) Optical oscillators. Researchers from the Optoelectronics Research Center (ORC) at the University of Southampton, UK, and Center for Disruptive Photonic Technologies (CDPT) at the Nanyang Technological University (NTU), Singapore, have demonstrated how neural dynamics in the brain can be simulated with optical pulses as information carriers using chalcogenide glass fibers that are sensitive to light [Gholipour, 2015]. The material of the fibers can switch from glass to a crystal and back again in response to varying wavelengths, intensities and polarizations of light. As with other photonic networks, neuromorphic devices could be built with the beneficial properties of ultra fast operation speeds, low thermal footprints and large bandwidth. The researchers are currently scaling the fibers to form much smaller waveguides and in the future it should be possible to fit hundreds of thousands of nodes on to a single chip.

(6) Biological neuronal oscillators. Finally, and most relevant to what will follow in this paper, biological neurons can be used to build binary oscillator logic circuits, memory devices and clocking circuits. Many researchers are attempting to build neuromorphic computers that will mimic the human brain, however, binary oscillator computing, although based on the dynamics of the brain, will be used to perform conventional computing. One of the potential applications of this technology is that it could be used to test functionality of new technologies and be used as a kind of half-way house to test whether or not the neurons and synaptic connections to be used in neuromorphic machines are performing in the correct way.

In Sections 2 and 3, we discuss the use of CMOS-based multi-electrode arrays (MEAs) for stimulating and recording from in vitro electrogenic cells, and we present the biophysically meaningful computational/mathematical models of neurons and chemical synapses which connect them. The results of simulations are shown in the Section 4 where we demonstrate full functionality of a neuron-based binary oscillator half-adder, a neuron-based SR flip-flop, and for the first time, a simple neuron-based clocking circuit. Simulations using biologically meaningful neural threshold oscillator models and chemical synaptic models demonstrate how such a model may be implemented even in the presence of noise. The final section presents a Discussion.

\section{Materials and methods}

We will now expand upon biological neuronal oscillator circuits, the main topic of this paper. A recent patent [Lynch, 2016] shows how in vitro cell assays, which employ binary half-adder and/or logic gates, can measure the effect a test condition has on cells and cell structures. The inventors are currently attempting to build a prototype of the neuronal assay and computational and mathematical modeling will aid in this construction. Figure 3(a) shows our apparatus set up where the computer monitor is displaying spike trains of neurons sat atop an MEA, and Figure 3(c) shows a close up of an MEA. 


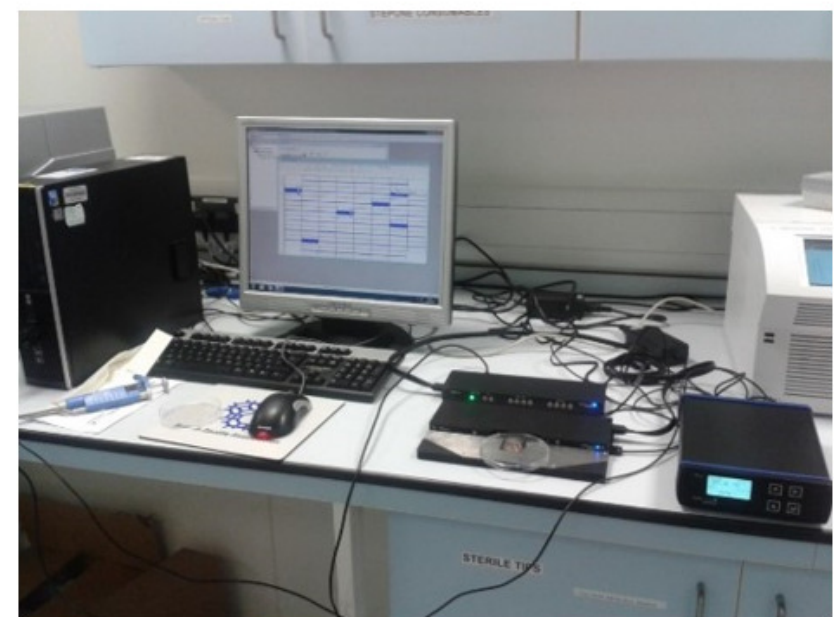

(a)

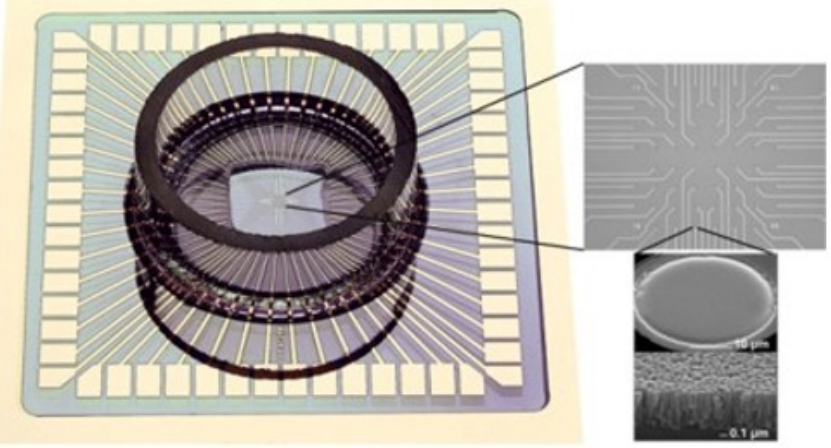

(c)

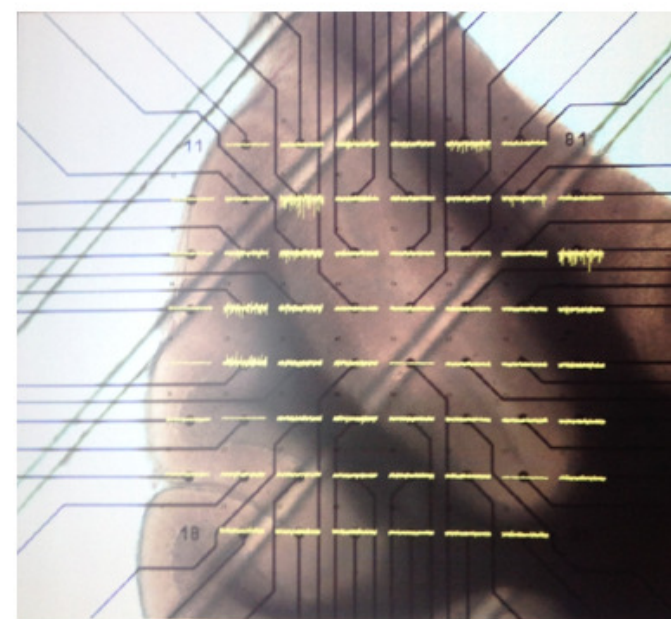

(b)

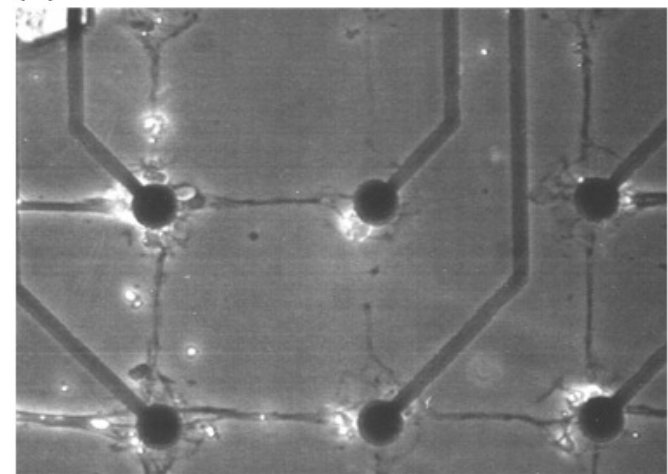

(d)

Fig. 3. Multi-Electrode Arrays (MEAs). (a) MEA apparatus - notice the spike trains on the computer monitor. (b) Brain slice on an MEA: The yellow time series traces that overlay the electrodes show the summative effect of surrounding neurons. (c) An MEA manufactured by Scientifica Ltd (http://www.scientifica.uk.com/). Used with permission of Scientifica Ltd. (d) Individual neurons on an MEA: Used with permission of C. Wyart.

In 1972, Thomas Jr. et al. [Pine, 2006] conducted the first experiments incorporating the use of planar electrodes on an MEA to record electrical activity from cultured myocyte cells. A sharp change in voltage in the extracellular environment, usually caused by an ion influx through the cellular membrane, can be detected on an MEA connected to a computer and these readings are being used extensively to characterize both network and cellular activity. The technology and related culture techniques for both tissue and electro-physiological cell assays have been greatly improved in recent years. High spatio-temporal resolution and low noise recordings have been taken from retinal cells [Wong, 1993], heart cells [Mauritz, 2008], mammalian spinal neuronal cells [Gross, 1995], muscle cells [Peng, 2001], brain cells [Cohen, 2011], and MEAs can be used in drug discovery and basic research [Stett, 2003]. Acute tissue preparations such as brain slices [Egert, 1998], see Fig 3(b), for example, enable immediate recording after removal from the animal and in-vivo positions remain intact. In this case, simultaneous multi-site long-term recording is required and these systems provide useful tools to measure synaptic plasticity, neural networks and pathophysiological conditions such as ischemia, Alzheimer's disease and epilepsy. Coating the MEA chips with cell-adhesion substrates such as collagen, fibronectin, laminin and poly-D-lysine aid adhesion of the cells or tissue. In 2005, Wyart et al. [Wyart, 2005] introduced a new technique to control the architecture of networks of individual neurons in vitro. Fig 3(d) (used with Wyart's permission) shows individual neurons situated in close proximity to electrodes with connecting neurites (or axons) following chemical trails to connecting neurons. In 2010, Wheeler and Brewer [Wheeler, 2010] provided an overview of the lithographic techniques used to attach neurons and guide axonal growth to form networks. They highlight five micro- 
patterning technologies for cellular lithography including laser ablation, micro-contact printing, microchannel deposition, micro-fluidic deposition and photo-resist patterning. Materials that promote cell growth include various amino silanes, laminin and polylysine, while albumin, chondroitin sulfate and polyethylene glycol, for example, restrict cell growth. The authors also state that baseline activity can be manipulated using changes in media composition, stimulation and glial addition, for example. In what is to follow we will present mathematical models of single connected neurons, whilst this is possible using current technology, activity, survivability and function are difficult at this stage [Khatami, 2008]. However, Chang et al. [Chang, 2001] showed that when local cell densities exceeded 250 cells per $\mathrm{mm}^{2}$, there was substantial activity and longevity of cell function. Using platforms consisting of chambers and tunnels [Campenot, 1977; Park, 2008] will offer tremendous advantages as each chamber can be filled with a cluster of excitatory or inhibitory cells, directed axonal growth can be achieved and a variety of local geometries (even three-dimensional) is possible. Action potentials can be monitored at the source, along the communication channel and at the target. Using a nano-material bio-screening platform for neurological applications [Jenkins, 2015], the authors are currently working with Dr Paul Roach, Loughborough University, in an attempt to build logic and memory circuits using clusters of neurons in chambers connected by tunnels. These results will be published at a later date. Three-dimensional MEAs for recording dissociated neuronal cultures have been developed [Edelman, 2005; Musick, 2009] and more recently, Lozano et al. [Lozano, 2015] have bioprinted three-dimensional layered brain-like structures using peptide modified gellan gum substrates allowing for more accurate in vitro micro-structures.

In 2002, Zemelman et al. [Zemelman, 2002] developed a method for stimulating genetically modified neurons using light and this has led to the field of research known as optogenetics. The field of optogenetics is quickly moving beyond proof of concept and is finding applications in cell signaling, biophysical modeling and systems biology, see [Tischer, 2014] for a review up to 2014. In 2008, Basu et al. [Basu, 2008] developed a new strategy to selectively label excitatory and inhibitory neurons in the cerebral cortex of mice. In 2015, Fosque et al. [Fosque, 2015] showed that a fluorescent protein-based reagent could permanently mark active populations of neurons in vivo over short time scales. In the future, with the developments in optogenetics and fluorescent marking it should be possible to develop our logic, memory and clocking circuits without the need for electronic intervention.

\section{Mathematical models}

\section{The Hodgkin-Huxley model: A biophysically meaningful model}

For the first time, we present biophysically realistic models of a biological half-adder, an SR flip-flop and a clocking circuit using the famous Hodgkin and Huxley equations [Hodgkin, 1952]. We present the differential equations for completeness:

$$
I=C \frac{d V}{d t}+I_{N a}+I_{K}+I_{L}
$$

where $I$ is the total membrane current density, $C$ is the membrane capacitance per unit area, $V$ is the difference between the membrane potential and the resting potential, $I_{N a}$ is the sodium current, $I_{K}$ is the potassium current, and $I_{L}$ is the leakage current. Hodgkin and Huxley were able to expand equation(1) to give:

$$
C \frac{d V}{d t}=I-g_{N a} m^{3} h\left(V-V_{N a}\right)-g_{K} n^{4}\left(V-V_{K}\right)-g_{L}\left(V-V_{L}\right),
$$

where $V_{N a}, V_{K}, V_{L}, C$ and $g_{L}$ are all constants determined from experimental data, and $g_{N a}$ and $g_{K}$ are the sodium and potassium conductances per unit area, respectively. The three dimensionless quantities $m$, $h$, and $n$ represent sodium, potassium and leakage gating variables and evolve according to the differential 
equations:

$$
\begin{aligned}
& \frac{d m}{d t}=\alpha_{m}(1-m)-\beta_{m} m \\
& \frac{d h}{d t}=\alpha_{h}(1-h)-\beta_{h} h \\
& \frac{d n}{d t}=\alpha_{n}(1-n)-\beta_{n} n,
\end{aligned}
$$

where $\alpha_{i}$ and $\beta_{i}$ are the transition rate constants for the $i$-th ion channel. The individual gates act in a similar manner to first order chemical reactions with two states. The rate constant $\alpha_{i}$ represents the number of times per second that a shut gate opens, and similarly, $\beta_{i}$ represents the number of times per second that an open gate shuts.

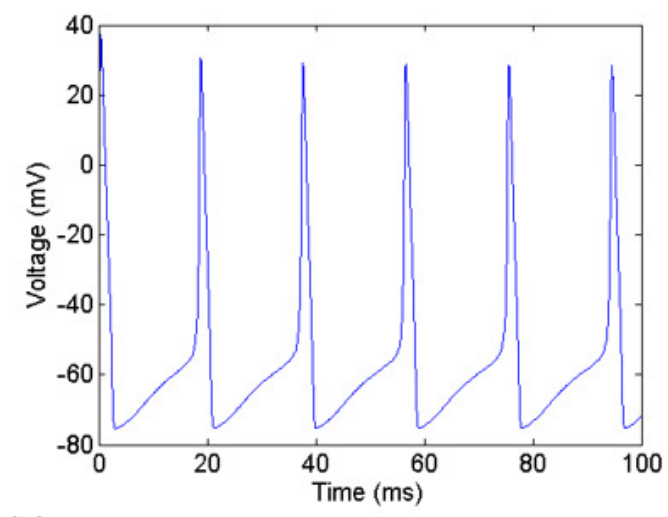

(a)
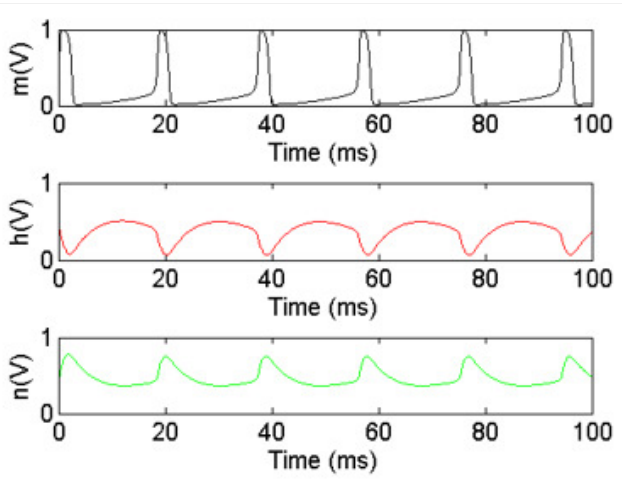

(b)
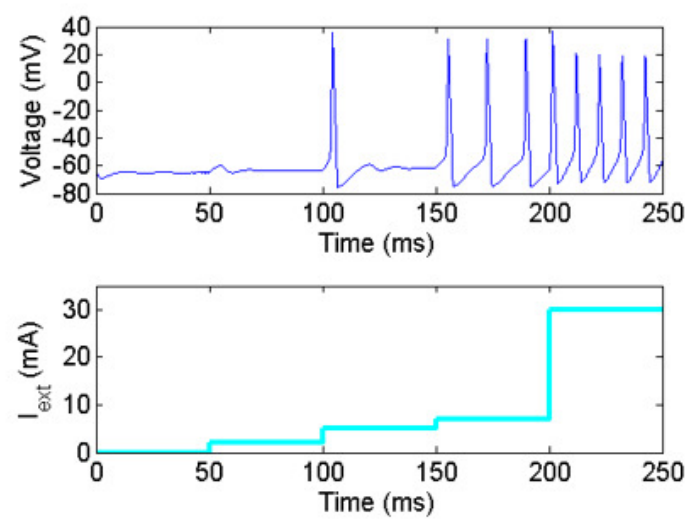

(c)

Fig. 4. Solutions of the Hodgkin-Huxley equations. (a) Spike train of action potentials that travel down the axon. At the beginning of the action potential the $\mathrm{Na}^{+}$channels open and $\mathrm{Na}^{+}$ions move into the axon causing depolarization. Re-polarization occurs when the $\mathrm{K}^{+}$channels open and $\mathrm{K}^{+}$ions move out of the axon. The signal travels down the axon to the axon terminal where it can trigger other neurons. (b) The gating variables $m, h$, and $n$ for equations and parameters listed in equations (2), (3), (4), and (5). (c) Neuron membrane voltage (upper blue trace) subject to an external input current $I_{\text {ext }}$ (lower cyan trace). As the input current is increased, the neuron eventually forms a spike train and the frequency of oscillation increases up to some physical limit.

Based on experimental data, the following parameter values have been chosen to generate Figures 4(a) 
and 4(b):

$$
\begin{aligned}
\alpha_{m} & =\frac{0.1(V+40)}{1-\exp (-0.1(V+40))}, \quad \beta_{m}=4 \exp (-0.0556(V+65)), \\
\alpha_{h} & =0.07 \exp (-0.05(V+65)), \quad \beta_{h}=\frac{1}{1+\exp (-0.1(V+35))}, \\
\alpha_{n} & =\frac{0.01(V+55)}{1-\exp (-0.1(V+55))}, \quad \beta_{n}=0.125 \exp (-0.0125(V+65)),
\end{aligned}
$$

and additionally,

$$
\begin{aligned}
C & =1 \mu \mathrm{Fcm}^{-2}, \\
g_{L} & =0.3 \mathrm{~ms} / \mathrm{cm}^{2}, g_{K}=36 \mathrm{~ms} / \mathrm{cm}^{2}, g_{N a}=120 \mathrm{~ms} / \mathrm{cm}^{2}, \\
V_{L} & =-54.402 \mathrm{mV}, V_{K}=-77 \mathrm{mV}, V_{N a}=50 \mathrm{mV} .
\end{aligned}
$$

In the simplest sense, neurons are either firing or not firing. Once the neuron has been sufficiently excited above some threshold (typically $-55 \mathrm{mV}$ in human neurons), the cell fires, if the neuron does not reach this threshold, it will not depolarize or create an action potential. If the stimulus does not reach threshold, then the neuron does not fire. As the stimulus passes the threshold value and continues to rise, the neuron starts to fire and the amplitude of oscillation remains constant, hence the All or None principle of neuron firing. Note, however, as the stimulus increases the frequency of oscillation increases up to some physical limit. Figure 4(c) illustrates each of these outcomes, the lower figure shows the external input current to the neuron and the upper figure shows the corresponding membrane voltage. When the external input current is zero $(0 \leq t \leq 50 \mathrm{~ms})$, the neuron does not fire. When the input current is increased to $2 \mathrm{mV}$ $(50 \leq t \leq 100 \mathrm{~ms})$, the membrane voltage rises slightly, but the neuron still does not fire. When the input current is raised to $5 \mathrm{mV}(100 \leq t \leq 150 \mathrm{~ms})$, the neuron spikes once and returns to its resting state. When the input current reaches $7 \mathrm{mV}(150 \leq t \leq 200 \mathrm{~ms})$, the neuron starts to spike at regular intervals and as the input current increases, the frequency of oscillation increases up to a physical limit and then switches off again.

In order to model chemical excitation and inhibition, Hodgkin-Huxley ODEs are coupled together that model the membrane potential in both presynaptic and postsynaptic neurons, and both the excitatory and inhibitory synaptic currents are modeled using models of chemical kinetics.

The next section shows how the chemical kinetics are modeled with differential equations which are again included for the sake of completeness.

\section{Mathematical model of biophysically meaningful chemical synapses}

In 1994, Destexhe et al. [Destexhe, 1994] derived an efficient method for computing synaptic conductances based on chemical kinetics. As an action potential reaches a presynaptic axon terminal, neurotransmitter molecules, $T$ say, are released and diffuse across the synaptic gap to bind with specific receptors in the postsynaptic neuron. This binding mechanism can be modeled using the stoichiometric equation:

$$
\mathrm{R}+\mathrm{T} \rightleftharpoons \mathrm{RT},
$$

where $[T]$ is the concentration of neurotransmitter, $[R]$ is the proportion of concentration of unbound receptor, $[R T]$ is the proportion of concentration of bound receptor, and $k_{f}$ and $k_{r}$ are the forward and reverse rate constants, respectively. Suppose that $B=[R T]$, then

$$
\frac{d B}{d t}=k_{f}[T](1-B)-k_{r} B
$$

Often in mathematics we have to make assumptions and simplifications. It is important to note at this stage that it is an oversimplification to think of synapses in terms of only one neurotransmitter. In reality, more then one neurotransmitter will diffuse across the synaptic gap to bind with specific receptors in the membrane of the adjoining neuron. In this case we will assume that the mediated excitatory receptor is N-methyl-D-aspartate (NMDA) and the mediated inhibitory receptor is $\gamma$-Aminobutyric acid (GABA). 
These are the most common excitatory and inhibitory neurotransmitters in the brain. There are others and some neurotransmitters, such as dopamine, can be both excitatory and inhibitory. However, the summative effect can be modeled (verified by experimentation [Destexhe, 1994]) using the following equations. Assuming that the neurotransmitter occurs as a pulse, then equation(7) leads to the following expressions:

1. During a pulse, when $t_{0}<t<t_{1},[T]=T_{\max }$ and $B$ is given by

$$
B\left(t-t_{0}\right)=B_{\infty}+\left(B\left(t_{0}\right)-B_{\infty}\right) \exp \left(\frac{-\left(t-t_{0}\right.}{\tau_{B}}\right),
$$

where

$$
B_{\infty}=\frac{k_{f} T_{\max }}{k_{f} T_{\max }+k_{r}}
$$

and

$$
\tau_{B}=\frac{1}{k_{f} T_{\max }+k_{r}} .
$$

2. After a pulse, when $t>t_{1},[T]=0$, and $B$ is given by

$$
B\left(t-t_{1}\right)=\left(B\left(t_{1}\right)\right) \exp \left(-k_{r}\left(t-t_{1}\right)\right) .
$$

In order to generate an excitatory conductance after a single transmitter pulse, the forward and reverse rate constants are set to $k_{f}=2 \mathrm{msec}^{-1} \mathrm{mM}^{-1}$ and $k_{r}=1 \mathrm{msec}^{-1} \mathrm{mM}^{-1}$. To generate an inhibitory conductance after a single transmitter pulse, the forward and reverse rate constants are set to $k_{f}=$ $0.5 \mathrm{msec}^{-1} \mathrm{mM}^{-1}$ and $k_{r}=0.05 \mathrm{msec}^{-1} \mathrm{mM}^{-1}$. With these parameter values, a single transmitter pulse will evoke a fast excitatory conductance which quickly decays or an inhibitory conductance which decays far more slowly. The excitatory and inhibitory synaptic currents, $I_{\mathrm{NMDA}}$ and $I_{\mathrm{GABA}}$, respectively, can then be written as

$$
I_{\mathrm{NMDA}}=G_{\mathrm{NMDA}} B_{\mathrm{NMDA}}(t)\left(V_{\mathrm{post}}(t)-E_{\mathrm{NMDA}}\right),
$$

and

$$
I_{\mathrm{GABA}}=G_{\mathrm{GABA}} B_{\mathrm{GABA}}(t)\left(V_{\mathrm{post}}(t)-E_{\mathrm{GABA}}\right),
$$

where $G_{\mathrm{NMDA}}, G_{\mathrm{GABA}}$ are the maximal conductances of the relevant synapse, $E_{\mathrm{NMDA}}, E_{\mathrm{GABA}}$ are the synaptic reversal potentials and $V_{\text {post }}$ is the post-synaptic potential. Thus from equation (1), for coupled neurons, the current in the postsynaptic neuron is given by

$$
I_{\mathrm{ext}}+I_{\mathrm{NMDA}}+I_{\mathrm{GABA}}=C \frac{d V}{d t}+I_{N a}+I_{K}+I_{L},
$$

where one or more of $I_{\mathrm{ext}}, I_{\mathrm{NMDA}}, I_{\mathrm{GABA}}$, could be zero.

\section{Results}

The results in this section were generated using MATLAB ${ }^{\circledR}$ and Mathematica ${ }^{\circledR}$, however, packages such as Maple ${ }^{\mathrm{TM}}$ and Python would produce the same results. Readers can reproduce these results by editing some of the programs listed in [Lynch, 2018, 2017, 2014, 2010].

Figure 5 shows the output for chemical excitation and inhibition using the Hodgkin-Huxley equations and the methods of Destexhe et al. Figure 5(a) shows the traces for a presynaptic neuron potential $V_{\text {pre }}$ (upper green trace), a ratio of excitatory conductance $B$ (middle magenta trace) and a postsynaptic neuron potential $V_{\text {post }}$ (lower blue trace). The transmitter pulse is initiated each time $V_{\text {pre }}$ exceeds a threshold of $0 \mathrm{mV}$, but only when the event function increases, that is the gradient of $V_{\text {pre }}$ has to be positive at this event. Referring to Figure 5(a), the first transmitter pulse is evoked when $t_{0}=18.557 \mathrm{~ms}, T_{\max }=1 \mathrm{mM}$, and the transmitter pulse duration $\left(t_{1}-t_{0}\right)=1 \mathrm{~ms}$. Subsequent transmitter pulses result in another four spikes in $B$. The lower trace $V_{\text {post }}$ shows that the neuron is at resting potential for $t_{0} \leq t \leq 20 \mathrm{~ms}$, but then starts to spike as a result of the excitatory synaptic current. Figure 5(b) shows the traces for a presynaptic 

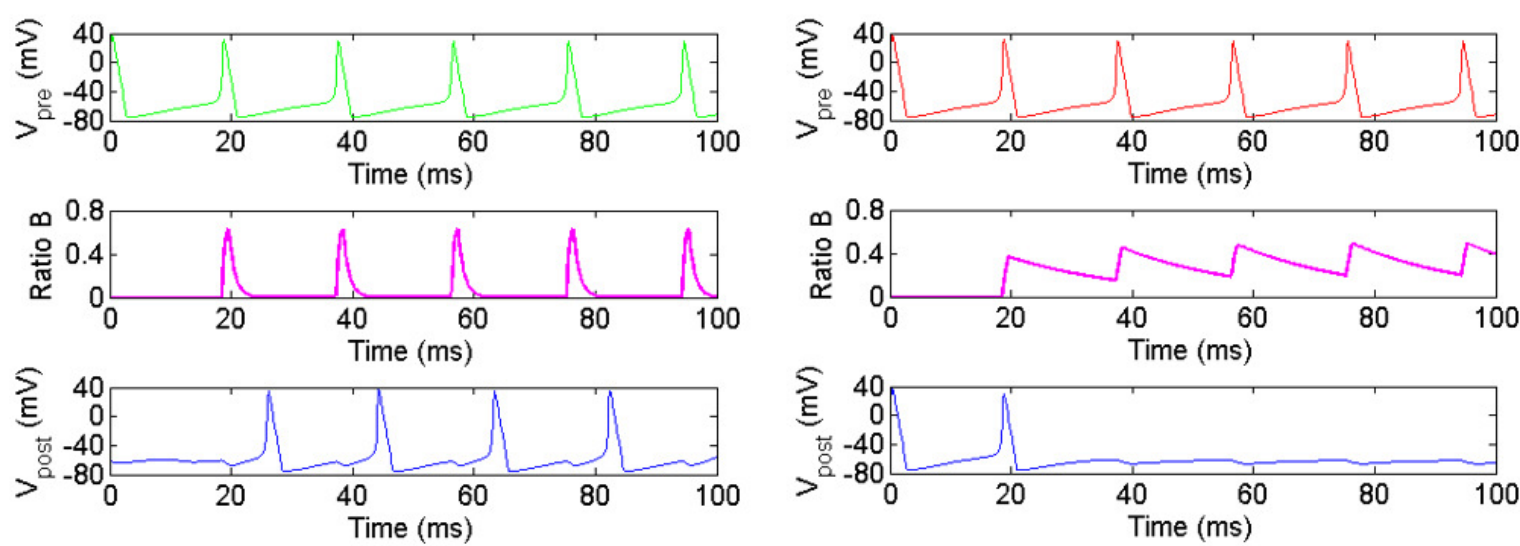

(a)

(b)

Fig. 5. Models of chemical excitation and chemical inhibition. (a) The upper figure shows the voltage of the presynaptic neuron voltage $V_{\text {pre }}$ (green curve) and the middle curve shows the ratio of excitatory conductance $B$. The lower curve shows the postsynaptic neuron voltage $V_{\text {post }}$, which is switched on by the excitatory synaptic current $I_{\text {NMDA }}$. The simulation was run using the parameters listed in equations (2)-(5) and (8)-(12) with $k_{f}=2 \mathrm{msec}^{-1} \mathrm{mM}^{-1}, k_{r}=1 \mathrm{msec}^{-1} \mathrm{mM}^{-1}$, $G_{\mathrm{NMDA}}=1 \mathrm{nS}$ and $E_{\mathrm{NMDA}}=0 \mathrm{mV}$. (b) The upper figure shows the voltage of the presynaptic neuron voltage $V_{\text {pre }}$ (red curve) and the middle curve shows the ratio of inhibitory conductance $B$. The lower curve shows the postsynaptic neuron voltage $V_{\text {post }}$, which is switched off by the inhibitory synaptic current, $I_{\mathrm{GABA}}$. The simulation was run using the parameters listed in equations (2)-(5) and (8)-(12) with $k_{f}=0.5 \mathrm{msec}^{-1} \mathrm{mM}^{-1}, k_{r}=0.05 \mathrm{msec}^{-1} \mathrm{mM}^{-1}, G_{\mathrm{GABA}}=1 \mathrm{nS}$ and $E_{\mathrm{GABA}}=-80 \mathrm{mV}$.

neuron potential $V_{\text {pre }}$ (upper red trace), a ratio of inhibitory conductance $B$ (middle magenta trace) and a postsynaptic neuron potential $V_{\text {post }}$ (lower blue trace). The transmitter pulse is initiated in the same way as for Figure 5(a), however, in this case an inhibitory synaptic current is evoked. Referring to Figure $5(\mathrm{~b})$, the first transmitter pulse is evoked when $t_{0}=18.557 \mathrm{~ms}, T_{\max }=1 \mathrm{mM}$, and the transmitter pulse duration $\left(t_{1}-t_{0}\right)=1 \mathrm{~ms}$. Subsequent transmitter pulses result in another four spikes in $B$. The lower trace $V_{\text {post }}$ shows that the neuron is spiking for $t_{0} \leq t \leq 20 \mathrm{~ms}$, but then is inhibited as a result of the inhibitory synaptic current.

Next we consider the simple biological neuronal circuitry for a half adder, an SR flip-flop and a simple clocking circuit, all subject to noise.

Figure 6(a) shows a schematic of a binary neuron-oscillator half adder connected to electrodes (shown in black) as it would appear on an MEA. Figure 6(b) shows the corresponding time series plot showing that the device is functioning correctly for a binary half adder when subjected to noise (see Figure 1(b)). The upper cyan plots show inputs $I_{1}$ and $I_{2}$, the green $V_{1}$ plot shows the output for neuron $N_{1}$ and the red $V_{2}$ plot shows the output for neuron $N_{2}$. The ratio $B$ magenta trace shows the ratio of inhibitory conductance $B$ and the lowest plot displays the noise in the circuit which is of a similar amplitude to the input.

Figure 7(a) shows a schematic of a binary neuron-oscillator SR flip-flop connected to electrodes (shown in black) as it would appear on an MEA. Figure 7(b) shows the corresponding time series plot showing that the device is functioning correctly as a binary SR flip-flop when subject to noise (see Fig 1(d)). The upper cyan plots show the input pulses $I_{1}$ and $I_{2}$, the outputs $V_{1}$ and $V_{2}$ in red are the outputs for neurons $N_{1}$ and $N_{2}$, respectively. The noise is displayed as the blue curve in the lowest plot and is of a larger magnitude than that of the input.

Finally, we present Figure 8(a) showing the schematic of a binary neuron-oscillator feedback clocking circuit as it would appear on an MEA. Figure 8(b) shows the time series voltage plots for neurons $N_{1}, N_{2}$, $N_{3}$ as $V_{1}, V 2, V_{3}$, respectively. In this case, neurons $N_{2}$ and $N_{3}$ are synchronized and out of phase with neuron $N_{1}$. This circuitry is also noise resistant.

Thus, the authors have demonstrated how all of the components required to build a computer, namely, ALUs, memory and clocking circuits could be implemented with biological neurons. Once these simple circuits have been constructed then it will be possible to build more complex logic, memory and clocking 

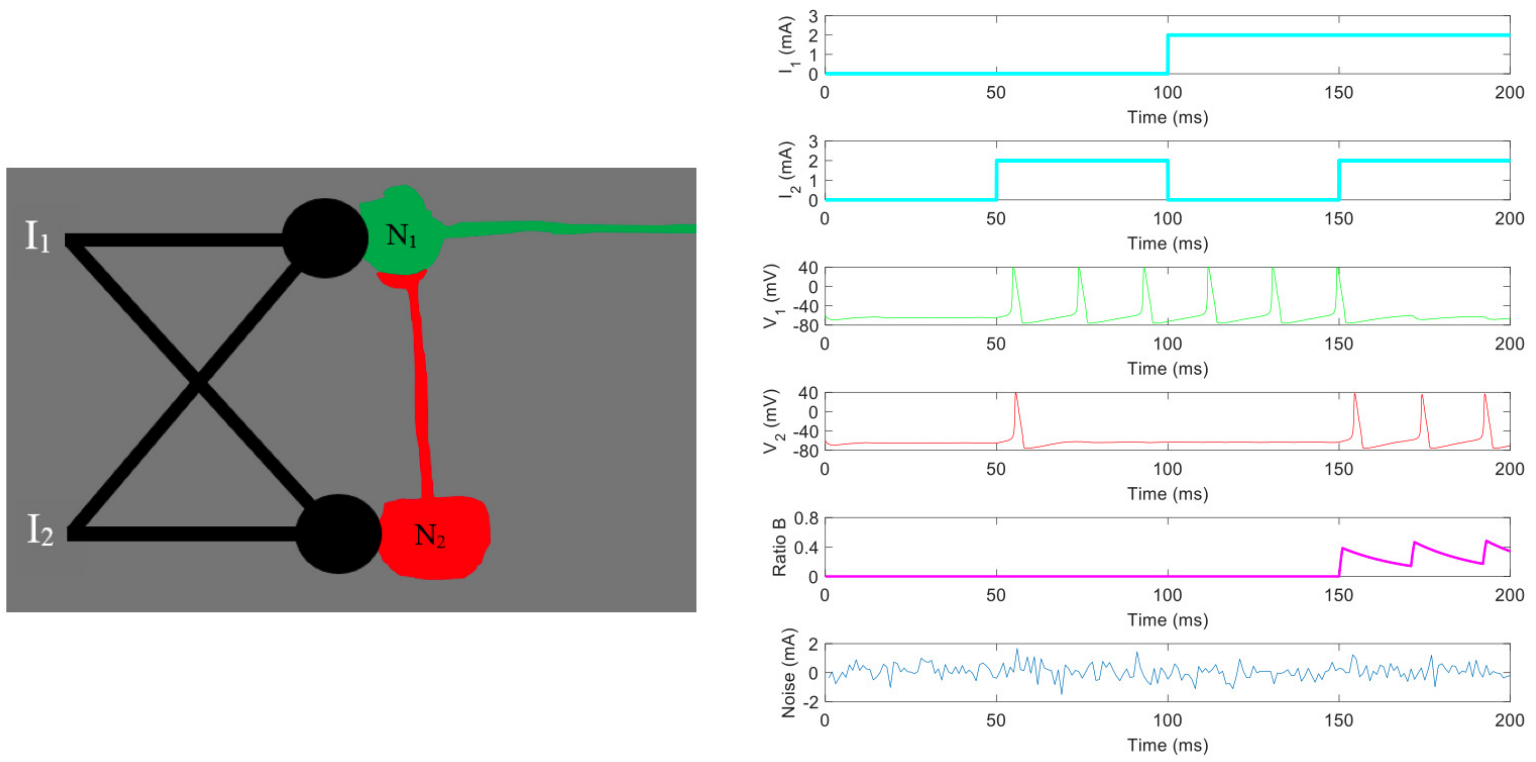

(a)

(b)

Fig. 6. Binary neuron threshold oscillator half adder. (a) Schematic of a binary neuron-oscillator half adder as it would look on an MEA. The excitatory neuron $N_{1}$ is colored green and the inhibitory neuron $N_{2}$ is colored red. (b) Time series showing correct functionality of a binary oscillator half-adder when subjected to noise. Refer to Figure 1(b). Note that in this case that there is an inhibitory synaptic connection to an excitatory neuron. The parameters used in this computational model were $g_{k}=36, g_{l}=0.3, V_{N a}=50, V_{K}=-77, V_{l}=-54.402$ and $C=1$. For the low threshold neuron $N_{1}, g_{N a}=170$ and for the high threshold neuron $g_{N a}=140$. The noise was generated using normally generated random numbers.

circuits. A typical neuron has only one axon, instead of requiring multiple axons, a neuron simply branches its axons via axon collaterals and in this way one neuron can connect with many other neurons (up to a thousand in the human brain) and most post-synaptic neurons receive the same signal. Propagation dynamics in branching axons are discussed in [Bakkum, 2013; Ofer, 2017]. However, due to the physical restrictions of biological neurons (mainly speed), building a conventional computer in this way would be impractical.

Implementation using one of the other technological paradigms mentioned in the introduction could be possible in the future or there may be bespoke applications of this research. Results of these investigations will be published at a later date.

\section{Discussion}

Implementation of the invention on binary oscillator computing can come from one of six technological paradigms - computational/mathematical models of transistor and Josephson junction have been discussed in [Lynch, 2012] and [Lynch, 2013], implementation in terms of memristors, superconducting nanowires and optical fibers is yet to be investigated. This paper concentrates on the biological aspect of the invention which could have far reaching implications in neural computing, reducing animal testing and drug research.

Our logic, memory and clocking circuits incorporate excitatory and inhibitory synaptic connections, inhibitory-inhibitory feedback synaptic connections and excitatory-inhibitory feedback connections. Readers may be interested in excitatory-excitatory feedback synaptic connections which are important in synchronization and de-synchronization dynamics, see for example, [Acker, 2003; Ashwin, 2004].

It is estimated that there are about 500 neurological conditions and disorders affecting the brain, spine and the nervous system. In 2005, the World Health Organization estimated that neurological disorders affected more than one billion people worldwide. Just one of these disorders, Alzheimer's disease (the most common form of dementia) currently has no cure and it is estimated that $1.2 \%$ of the world's population will be affected by 2050. In order to develop treatments for such disorders, it is essential to be able to 


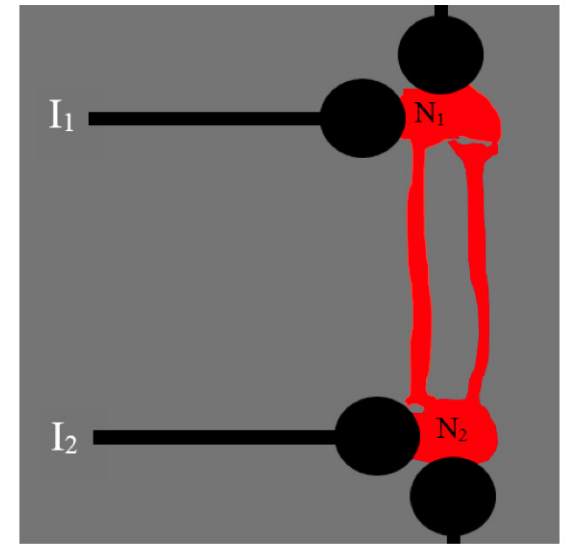

(a)
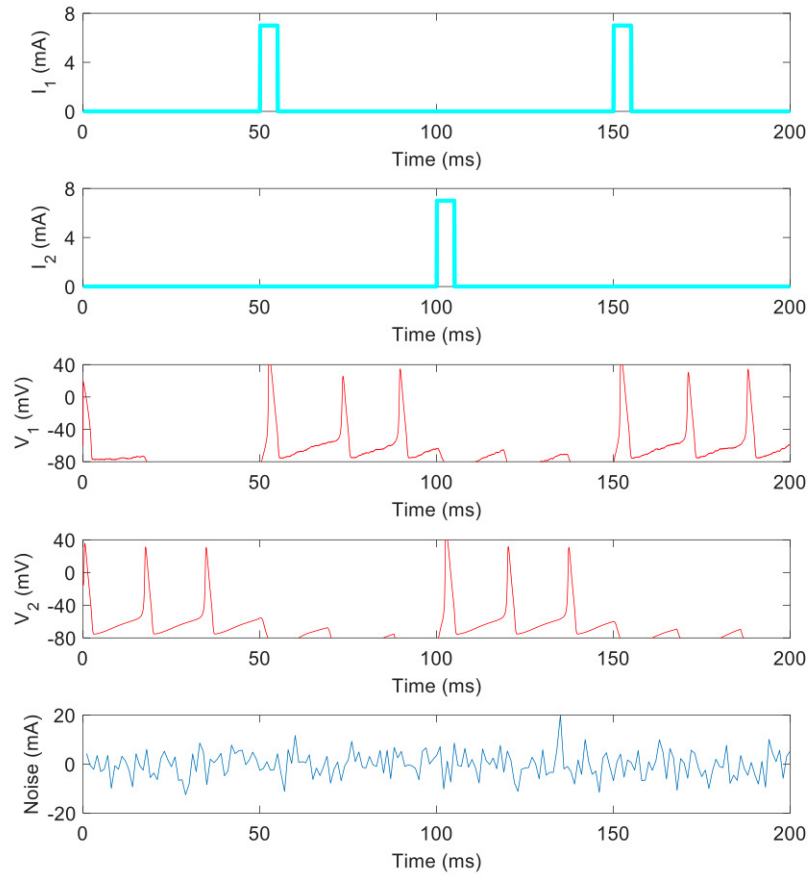

(b)

Fig. 7. Binary neuron threshold oscillator SR flip-flop. (a) Schematic of a binary neuron-oscillator SR flip-flop as it would look on an MEA. The inhibitory neurons $N_{1}$ and $N_{2}$ are colored red. (b) Time series showing correct functionality of a binary oscillator SR flip-flop when subjected to considerable noise. Refer to Fig 1(d). Note that in this case there are inhibitory-inhibitory feedback synaptic connections. The parameters used in this computational model were $g_{k}=36, g_{l}=0.3$, $V_{N a}=50, V_{K}=-77, V_{l}=-54.402$ and $C=1$. For both inhibitory neurons $N_{1}$ and $N_{2}, g_{N a}=120$ and $E_{G A B A}=120$. The noise was generated using normally generated random numbers.

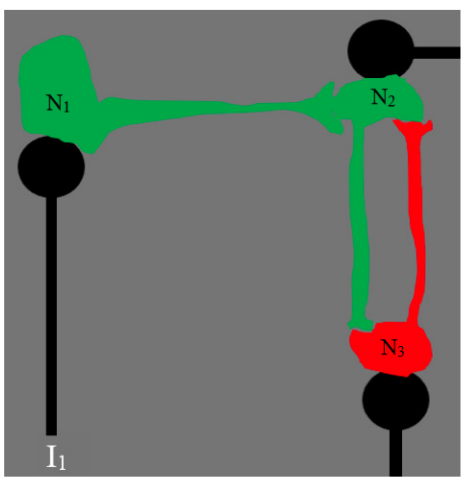

(a)

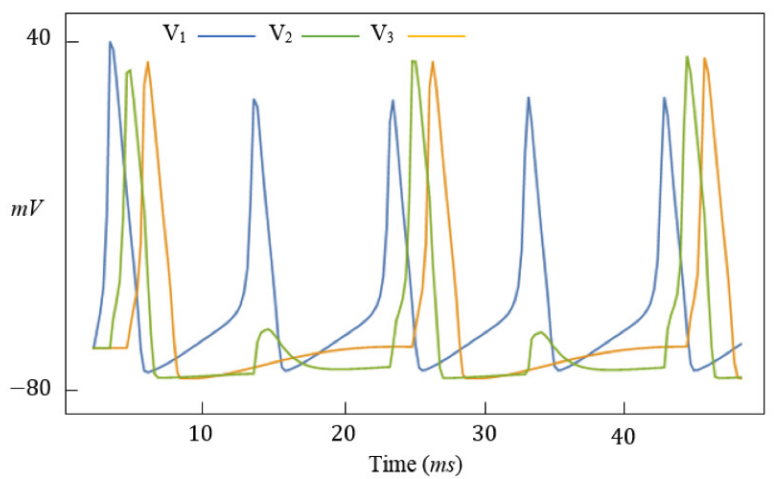

(b)

Fig. 8. Binary neuron threshold oscillator clocking circuit. (a) Schematic of a binary neuron-oscillator feedback clocking circuit as it would appear on an MEA. The excitatory neurons $N_{1}$ and $N_{2}$ are colored green and the inhibitory neuron $N_{3}$ is colored red. (b) Time series showing correct functionality of a binary neuron-oscillator clocking circuit. Note that in this case that there is an excitatory synaptic connection to an excitatory neuron and there are excitatory-inhibitory feedback synaptic connections. The parameters used in this computational model were $g_{k}=36, V_{N a}=50, V_{K}=-77, V_{l}=-54.402$ and $C=1$, for all neurons. Otherwise, $I_{1}=11, g 1_{N M D A}=5, g 2_{N M D A}=6, g 3_{G A B A}=8$. The noise was generated using normally generated random numbers. 
accurately assess the functionality of neural cells in-vitro before more invasive in-vivo tests are conducted. Growing neural cells atop multi-electrode arrays is now well established. These devices enable fundamental neurophysiological insights at both the circuit and cellular level. The advantages in using MEAs are welldocumented, see [Whitson, 2006], for example, and implementation of our circuitry should eventually be possible. Neurons are not the only oscillators in the human body, heart cells, muscle cells and retinal cells, for example, all oscillate and can excite and inhibit one another, and there are other oscillators that cycle on a wide range of time scales [Lynch, 2015]. The use of cardiac myocytes, for example, which are more robust than neurons, could provide alternative solutions to the problems that neurons bring with experimentation [Siso-Nadal, 2009]. It is also possible to set up co-cultures of sympathetic neurons and cardiac myocytes on MEAs [Lockhart, 1997]. Running several circuits simultaneously will increase the throughput and cut the costs of experimentation in drug research and since our circuits are so simple and can be replicated, it should be possible to test a number of drugs on one MEA.

Biophysical meaningful models of a binary oscillator half-adder, an SR flip-flop and a clocking circuit have been presented for the first time. There are several ways in which these ideas may be implemented, however, in the short term it appears that the use of fluidic chambers and tunnels may provide the most robust, reliable and durable assays using current technology. Dr Roach and his group are currently fabricating platforms enabling the segregated co-culture of neurons to be controlled, with connectivity between neuronal populations being possible only by direction of neurites through microchannels [Kamudzandu, 2019]. Such devices are becoming more commonplace in the literature, although very few address the issues of multiple different neuronal types within a single device. Further work is directed to humanizing this model and also to address specific challenges regarding the size of features used and their density. Chemical modification of MEAs will contribute to better control spatial location of cells for biological computing possibilities. When defining neuronal circuits down at the single-cell resolution, it is often difficult to maintain the viability of cells in such low density cultures. Furthermore, poisoning of multiple different neuronal sub-types within such a device raises issues of how to pattern these in mixed populations. One of the biggest drawbacks from the current work is the limited ability to replicate a humanized model. Many of the existing models rely heavily on the use of animal derived materials [Kose, 2017; Merryweather, 2017; Roach, 2008] which can be useful for biological computing applications but have limited scope within the area of drug testing or regenerative medicine. New approaches applying synthetic biology approaches to rapidly reprogram human pluripotent stem cells for the first time provide a robust and scalable source of functional human neurons [Pawlowski, 2017, 2013]. Neurons produced in this way display electro-physiological activity within less than two weeks and develop functional networks within three weeks [Tourigny, 2018].

Currently, the best assays in industry measure neurotoxicity via mean firing rate, burst characteristics and synchrony of spikes and bursts of clusters of neurons. They typically use stimulatory compounds, inhibitory compounds and seizurogenic compounds and then measure whether spike/burst rates have increased or decreased. Our assays, once manufactured, would provide the world with platforms which would provide far more detail than is available with current technology. By constructing neural circuits using our patented architecture, pharmaceutical companies would have an assay that measures the functionality of both neurons and their logic, memory and clocking circuits.

With regards to future patents, this technological area is increasingly of interest due to rapid growth in capabilities within the manufacture of biological-electrical hybrid devices. It is also becoming apparent that collaboration across the scientific disciplines is necessary to unlock the potential for future growth in this area, and that mathematical modeling will play a vital role in this endeavor.

\section{Acknowledgements}

We would like to take this opportunity to thank the referees for their extremely useful comments.

\section{References}

Acker CD, Kopell N, White JA [2003] Synchronization of strongly coupled excitatory neurons: Relating network behavior to biophysics, Journal of Computational Neuroscience 15(1): 7190. 
Afifi A, Ayatollahin A, Raissi F [2009] Implementation of Biologically Plausible Spiking Neural Network Models On the Memristor Crossbar-based CMOS/Nano Circuits, IEEE Conference: European Conference on Circuit Theory Design Location 1 and 2: 563-566.

Ashwin P, Borresen J [2004] Encoding via conjugate symmetries of slow oscillations for globally coupled oscillators, Physical Review E 70(2): 026203.

Bakkum DJ, Frey U, Radivojevic M et al. [2013] Tracking axonal action potential propagation on a highdensity MEA across hundreds of sites, Nature Communications 4: 2181.

Basu K, Gravel C, Tomioka R, Kaneko T, Tamamaki N, Sík A [2008] Novel strategy to selectively label excitatory and inhibitory neurons in the cerebral cortex of mice, J Neurosci. Methods 30: 212-9.

Borresen J, Lynch S [2012] Oscillatory threshold logic. PLoS ONE 7(11): e48498. doi:10.1371/journal.pone.0048498.

Borresen J, Lynch S [2009] Neuronal computers, Nonlinear Anal Theory, Meth and Appl, 71: 2372-2376.

Campenot RB [1977] Local control of neurite development be nerve growth-factor, Proc. Natl. Acad. Sci. USA 74: 4516-4519.

Chang JC, Brewer GJ, Wheeler BC [2001] Modulation of neural network activity by patterning, Biosens. Bioelectron. 16: 527-533.

Chua LO, Memristor, Hodgkin-Huxley and the edge of chaos [2013] Nanotechnology 24: 383001.

Chua LO, Memristor-missing circuit element, IEEE Transactions on Circuit Theory CT18, [1971], 507-519.

Cohen MR, Kohn A [2011] Measuring and interpreting neuronal correlations, Nature Neuroscience 14: 811-819.

Crotty P, Schult D, Segall K [2010] Josephson junction simulation of neurons, Phys Rev E 82: 011914.

Dana SK, Sengupta DC, Hu Chin Kun [2006] Spiking and bursting in Josephson junction, IEEE Transactions on Circuits and Systems II: Express Briefs 10: 1031-1034.

Destexhe A, Mainen ZF, Sejnowski TJ [1994] An efficient method for computing synaptic conductances based on a kinetic model of receptor binding, Neural Computation 6: 14-18.

Edelman DB, Keefer EW [2005] A cultural renaissance: In vitro cell biology embraces three-dimensional context, Exp. Neurol. 192: 1-6.

Egert U, Schlosshauer B, Fennrich S, Nisch W, Fejtl M, Knott T et al. [1998] A novel organotypic longterm culture of the rat hippocampus on substrate-integrated multi-electrode arrays, Brain Research Protocols 2: 229-242.

Filippov T, Sahua A, Kirichenkoa AF, Vernika IV, Dorojevets M, Ayalab CL et al. [2012] 20 GHz operation of an asynchronous wave-pipelined RSFQ arithmetic-logic unit, Physics Procedia 36: 59-65.

Filippov T, Dorojevets M, Sahu A, Kirichenko A, Ayala C, Mukhanov O [2011] 8-bit asynchronous wavepipelined RSFQ arithmetic-logic unit, IEEE Trans on Applied Superconductivity 21: 847-851.

Fosque BF, Sun Y, Dana H, Yang CT, Ohyama T, Tadross MR [2015] Labeling of active neural circuits in vivo with designed calcium integrators, Science 347: 755-760.

Gholipour B, Bastock P, Craig C, Khan K, Hewak D, Soci1 C [2015] Amorphous metal-sulphide microfibers enable photonic synapses for brain-like computing, Advanced Optical Materials 3: 635641.

Gross GW, Rhoades BK, Azzazy HME [1995] The use of neuronal networks on multielectrode arrays as biosensors, Biosensors and Bioelectronics 10: 553-567.

Hodgkin AL, Huxley AF [1952] A qualitative description of membrane current and its application to conduction and excitation in nerve, J. Physiol 117: 500-544. Reproduced in Bull Math Biol [1990] 52: $25-71$.

Indiveri G, Linares-Barranco B, Hamilton TJ, van Schaik A, Etienne-Cummings R, Delbruck T [2011] Neuromorphic silicon neuron circuits, Front Neuroscience 5: 1-23.

Jenkins SI, Roach P, Chari DM [2015] Development of a nanomaterial bio-screening platform for neurological applications, Nanomedicine-Nanotechnology Biology and Medicine 11: 77-87.

Jo SH, Chang T, Ebong I, Bhadviya BB, Mazumder P, Lu W [2010] Nanoscale memristor device as synapse in neuromorphic systems, Nano Letters 10: 1297-1301.

Josephson BD [1974] The discovery of tunnelling supercurrents, Reviews in Modern Physics 46: 251254.

Josephson BD [1962] Possible new effects in superconductive tunnelling, Physics Letters 1: 251-253.

Kamudzandu M, Köse-Dunn M, Evans MG, Fricker RA and Roach P [2019] A micro-fabricated in vitro 
complex neuronal circuit platform, Biomed. Phys. Eng. Express 5: 045016.

Kim KK, Gaba S, Wheeler D, Cruz-Albrecht JM, Hussain T, Srinivasa N et al. [2012] A Functional Hybrid Memristor Crossbar-Array/CMOS System for Data Storage and Neuromorphic Applications, Nano Lett. 12: 389-395.

Khatami D [2008] Considerations for electrical stimulation and functional connectivity analysis of neuronal networks patterned on micro-electrode arrays, Ph.D. thesis, University of Illinois, Urbana.

Köse-Dunn M, Fricker R, Roach P [2017] Tissue engineered organoids for neural network modelling, Adv. Tissue Eng. Regen. Med. 3(3), 00066.

Linn E, Siemon A, Waser R, Menzel S [2014] Applicability of well-established memristive models for simulations of resistive switching devices, Circuits and Systems I: Regular Papers, IEEE Transactions on 61: 24022410.

Lockhart ST, Turrigiano GG, Birren SJ [1997] Nerve growth factor modulates synaptic transmission between sympathetic neurons and cardiac myocytes, The Journal of Neuroscience 17: 9573-9582.

Lozano R, Stevens L, Thompson BC, Gilmore KJ, Gorkin R, Stewart EM, et al. [2015] 3-D printing of layered brain-like structures using peptide modified gellan gum substrates, Biomaterials 67: 264-73.

Lynch S [2018] Dynamical Systems with Applications using Python, Springer International Publishing, Switzerland.

Lynch S [2017] Dynamical Systems with Applications using Mathematica 2nd Ed., Springer International Publishing, Switzerland.

Lynch S, Borresen J, Slevin MA [2016] International patent: Cell assays, development of binary half-adder. Publication Number US 2015/0276707 A1.

Lynch S, Borresen J [2015] Oscillations, feedback and bifurcations in mathematical models of angiogenesis and haematopoiesis, in Handbook of Vascular Biology Techniques, Slevin M, McDowell G, Cao Y, Kitajewski J eds., Springer, New York, 373-390.

Lynch S [2014] Dynamical Systems with Applications using MATLAB 2nd Ed., Springer International Publishing, Switzerland.

Lynch S, Borresen J, Latham K [2013] Josephson junction binary oscillator computing, Proceedings of the IEEE 14th International Superconductive Electronics Conference (ISEC) Cambridge, Massachusetts.

Lynch S, Borresen J [2012] Binary Half Adder using Oscillators, International Publication Number, WO 2012/001372 A1: 1-57.

Lynch S [2010] Dynamical Systems with Applications using Maple 2nd Ed., Springer International Publishing, Switzerland.

Mauritz C, Schwanke K, Reppel M, Neef S, Katsirntaki K, Maier LS et al. [2008] Generationof functional murine cardiac myocytes from induced pluripotent stem cells, Circulation 118: 507-517.

Merolla PA, Arthur JV, Alvarez-Icaza1 R, Cassidy AS, Sawada J, Akopyan1 F, et al. [2014] A million spiking-neuron integrated circuit with a scalable communication network and interface, Science 8: 668-673.

Merryweather D, Roach P [2017] The need for advanced three-dimensional neural models and developing enabling technologies, MRS Communications 7(3): 309-319.

Mizugaki Y, Nakajima K, Sawada Y, Yamashita T [1993] Superconducting neural circuits using fluxon pulses.

Mukhanov O [2011] Energy-efficient Single Flux Quantum technology, IEEE Trans Appl Supercond 21:760769.

Mukhanov O, Semenov VK [1988] Reproduction of the Single Flux Quantum pulses in Josephson junction systems. II. Neuristor lines and logic elements. Mikroelektronika [Sov. Microelectronics] 17:155-161.

Mukhanov OA, Semenov VK, Likharev KK [1987] Ultimate performance of the RSFQ Logic Circuits, IEEE Trans Mag 23: 759-762.

Musick K, Khatami D, Wheeler BC [2009] Three-dimensional micro-electrode array for recording dissociated neuronal cultures, Lab Chip 9: 2036-2042.

Ofer N, Shefi O and Yaari G [2017] Branching morphology determines signal propagation dynamics in neurons, Sci Rep. 7: 8877.

Ohno T, Hasegawa T, Tsuruoka T, Terabe K, Gimzewski JK, Aono M [2011] Short-term plasticity and 
long-term potentiation mimicked in single inorganic synapses, Nature Materials 10: 591-595.

Papandroulidakis G, Serb A, Khiat A, Merrett G and Prodromakis T [2019] Practical implementation of memristor-based threshold logic gate, IEEE Transactions on Circuits and Systems I: Regular Papers 66: $1-12$.

Park JW, Vihadi B, Kim HJ, Rhee SW, Jeon NL [2008] Quantitative analysis of CNS axon regeneration using a microfluidic neuron culture device, BioChip J. 2 :44-51.

Pawlowski M, Ortmann D, Bertero A, Tavares JM, Pederson RA, Vallier L, Kotter MRN [2017] Inducible and deterministic forward programming of human pluripotent stem cells into neurons, skeletal myocytes, and oligodendrocytes, Stem Cell Reports 8(4): 1-10.

Pawlowski M, Kotter MR [2013] Generation of neural cells by direct cellular reprogramming, Journal of Transplant Stem Cell Biology 1(1): 7.

Peng HL, Matchkov V, Ivarsen A, Aalkjaer C, Nilsson H [2001] Hypothesis for the initiation of vasomotion, Circulation Research 88: 810-815.

Peplow M (Editor) [2018] Blackett Review, Computational Modelling: Technological Futures, Government Office for Science and Council for Science and Technology,

URL: https://www.gov.uk/government/publications/computational-modelling-blackett-review [last accessed 8th July 2019].

Pickett MD, Medeiros-Ribeiro G, Williams RS, [2013] A scalable neuristor built with Mott memristors, Nature Materials 12: 114.

Pine J, A History of MEA Development. In: Baudry M, Taketani M, eds. Advances in Network Electrophysiology Using Multi-Electrode Arrays. New York: Springer Press [2006]: 3-23.

Roach P, Shirtcliffe NJ, Newton MI [2008] Progress in superhydrophobic surface development, Soft Matter 4(2): 224-240.

Ryazanov V, Bol'ginov V, Sobanina DS, Vernikc IV, Tolpygoc SK, Kadinc AM, et al. [2012] Magnetic Josephson junction technology for digital and memory applications, Physics Procedia 36:35-41.

Segall K, LeGro M, Kaplan S et al. [2017] Synchronization dynamics on the picosecond timescale in coupled Josephson junction neurons, Phys. Rev. E. 95: 032220.

Siso-Nadal F, Fox JJ, Laporte SA, Hebert TE, Swain PS [2009] Cross-talk between signaling pathways can generate robust oscillations in calcium and cAMP, PLoS One 4: e7189.

Stett A, Egert U, Guenther E, Hofmann F, Meyer T, Nisch W [2003] Biological application of microelectrode arrays in drug discovery and basic research, Analytical and Bioanalytical Chemistry 377: 486-495.

Strukov DB, Snider GS, Stewart DR, et al. [2008] The missing memristor found, Nature 453: 80-83.

Tischer D, Weiner OD [2014] Illuminating cell signaling with optogenetic tools, Nature Reviews Molecular Cell Biology 15: 551-558.

Toomey E, Segall K, Berggren KK [2019] Design of a power efficient artificial neuron using superconducting nanowires, Frontiers of Nanoscience 13:933: 1-12.

Tourigny DS, Karim MKA, Echeveste R, Kotter MRN, ONeill JS [2018], Energetic substrate availability regulates synchronous activity in an excitatory neural network, bioRxiv, 10.1101/363671.

URL: https://www.biorxiv.org/content/10.1101/363671v2.article-info [last accessed 8th July 2019].

Wheeler BC, Brewer GJ [2010] Designing neural networks in culture, Proceedings of the IEEE 98: 398-406.

Whitson J, Kubota D, Shimono K, Jia Y, Taketani M [2006] Multi-Electrode Arrays: Enhancing traditional methods and enabling network physiology. In: Baudry M, Taketani M, eds. Advances in Network Electrophysiology Using Multi-Electrode Arrays. New York: Spring Press: 38-68.

Wong ROL, Meister M, Shatz CJ [1993] Transient period of correlated bursting activity during development of the mammalian retina, Neuron 11: 923-938.

Wyart C, Ybert C, Douarche C, Herr C, Chatenay D, Bourdieu L [2005] A new technique to control the architecture of neuronal networks in vitro, Poindron P, Piguet P, Förster E (eds): New Methods for Culturing Cells from Nervous Tissues. BioValley Monogr. Basel, Karger, 1: 2357.

Zemelman BV, Lee GA, Ng M, Miesenbock G [2002] Selective photostimulation of genetically ChARGed neurons, Neuron 33: 15-22.

Zhou Y, Ramanathan S [2015] Mott memory and neuromorphic devices, Proceedings of the IEEE 103: 12891310. 